\title{
Aspergillus niger bloodstream infection in gastric cancer after common hepatic artery embolization: A case report
}

\author{
LI LIN $^{1}$, CHUAN-HUA ZHAO $^{1}$, XIU-YUN YIN $^{2}$, YU-LING CHEN $^{1}$, \\ HONG-YAN ZHAI ${ }^{3}$, CHUN-WEI XU ${ }^{4}$, YAN WANG ${ }^{1}$, FEI-JIAO GE ${ }^{1}$ and JIAN-MING XU ${ }^{1}$ \\ ${ }^{1}$ Department of Gastrointestinal Oncology, Affiliated Hospital Cancer Center; Departments of ${ }^{2}$ Clinical Laboratory, \\ ${ }^{3}$ Infection Control and ${ }^{4}$ Pathology, Affiliated Hospital, Academy of Military Medical Sciences, Beijing 100071, P.R. China
}

Received July 29, 2016; Accepted May 5, 2017

DOI: $10.3892 / \mathrm{etm} .2017 .4693$

\begin{abstract}
The present case study reported on a 62-year-old male patient with gastric cancer-associated Aspergillus (A.) niger bloodstream infection. The patient presented with massive hemorrhage in the gastrointestinal tract 3 months after total gastrectomy for gastric cancer. Conservative treatment consisting of blood transfusion to supplement blood volume loss was ineffective. Digital subtraction angiography indicated gastroduodenal artery bleeding. The first attempt of performing arterial embolization using gelatin sponges failed, while the second attempt of performing common hepatic artery embolization using gelatin sponges and micro-coil springs stopped the bleeding. Four weeks after angiography, the patient presented with the complication of $A$. niger bloodstream infection, which was cured using intravenous and oral voriconazole. Clinicians should be aware of the possibility of A. niger bloodstream infection after invasive operations in immunocompromised patients and apply timely antifungal treatment.
\end{abstract}

\section{Introduction}

With the incidence of cancer on the rise and the increasing use of immunosuppressant in recent years, Aspergillus infection has become the second most prevalent deep fungal infection following monilial infection $(1,2)$. Aspergillus bloodstream infection (BSI) is a rarely seen critical illness in the clinic, and most cases reported in the literature have dissemination of invasive pulmonary aspergillosis and end-stage infection in critical patients (3-5), with few patients having infection without organ dissemination. The present study reported on a

Correspondence to: Professor Jian-Ming Xu, Department of Gastrointestinal Oncology, Affiliated Hospital Cancer Center, Academy of Military Medical Sciences, 8 Dongda Street, Fengtai, Beijing 100071, P.R. China

E-mail: jmxu2003@yahoo.com

Key words: gastroduodenal artery bleeding, common hepatic artery embolization, Aspergillus niger, bloodstream infection case of gastric cancer with massive hemorrhage in the gastrointestinal tract, and the patient developed Aspergillus niger (A. niger) BSI after common hepatic artery (CHA) embolization.

\section{Case study}

A 62-year-old man with intermittent abdominal pain and $400 \mathrm{ml}$ of hematochezia was admitted to the Department of Emergency of the Affiliated Hospital of the Academy of Military Medical Sciences (Beijing, China) on March 13, 2012. After admission, the patient experienced dizziness, but without hematemesis.

The patient had received a total gastrectomy under general anesthesia for gastric cancer at Peking University People's Hospital (Beijing, China) on December 9th, 2011. Post-operative pathology had revealed moderately- and poorly-differentiated adenocarcinoma invasion in gastric tissue (gastric angle and cardia). Part of the lesion was manifested as mucinous adenocarcinoma $(3.7 \times 3.2 \times 0.5 \mathrm{~cm})$, and the other part of it was differentiated into signet ring cell carcinoma $(4 \times 3 \times 1 \mathrm{~cm})$. The tumor invaded the entire peritoneum and surrounding fat. Vascular tumor thrombus was visible, with negative upper and lower margins. Lymph node metastasis occurred in the greater and lesser curvature of the stomach (5/23 and 6/19), and metastatic carcinoma was seen in the lymph nodes submitted for detection $(12 \mathrm{~A} ; 1 / 1)$. There was no tumor invasion in fibrofatty tissue (12P) or the greater omentum. Immunohistochemical analysis revealed the following: Creatine kinase(-), cytokeratin 20(+), CDX2(+++), villin(++), Ki-67(50\%+), P53(++), CerbB-2(-), glycoprotein hormones, alpha polypeptide(-), synaptophysin (Syn)(-) and CD56(-). Specific alcian blue/periodic acid Schiff staining was positive on December 12, 2011.

The patient received paclitaxel $[150 \mathrm{mg}$ day (d)1, $120 \mathrm{mg} \mathrm{d}$ ], oxaliplatin (200 mg d1) plus Xeloda [1,500 mg twice a day (bid) d1-d14] from February 7, 2012 following strength recovery, which enables individuals to withstand chemotherapy, as a post-operative adjuvant chemotherapy for the first cycle. The paclitaxel scheme was withdrawn on March 1, 2012 during the second cycle due to self-reported fatigue that could not be tolerated. Physical examination revealed a body temperature of $36.8^{\circ} \mathrm{C}$, a heart rate of 


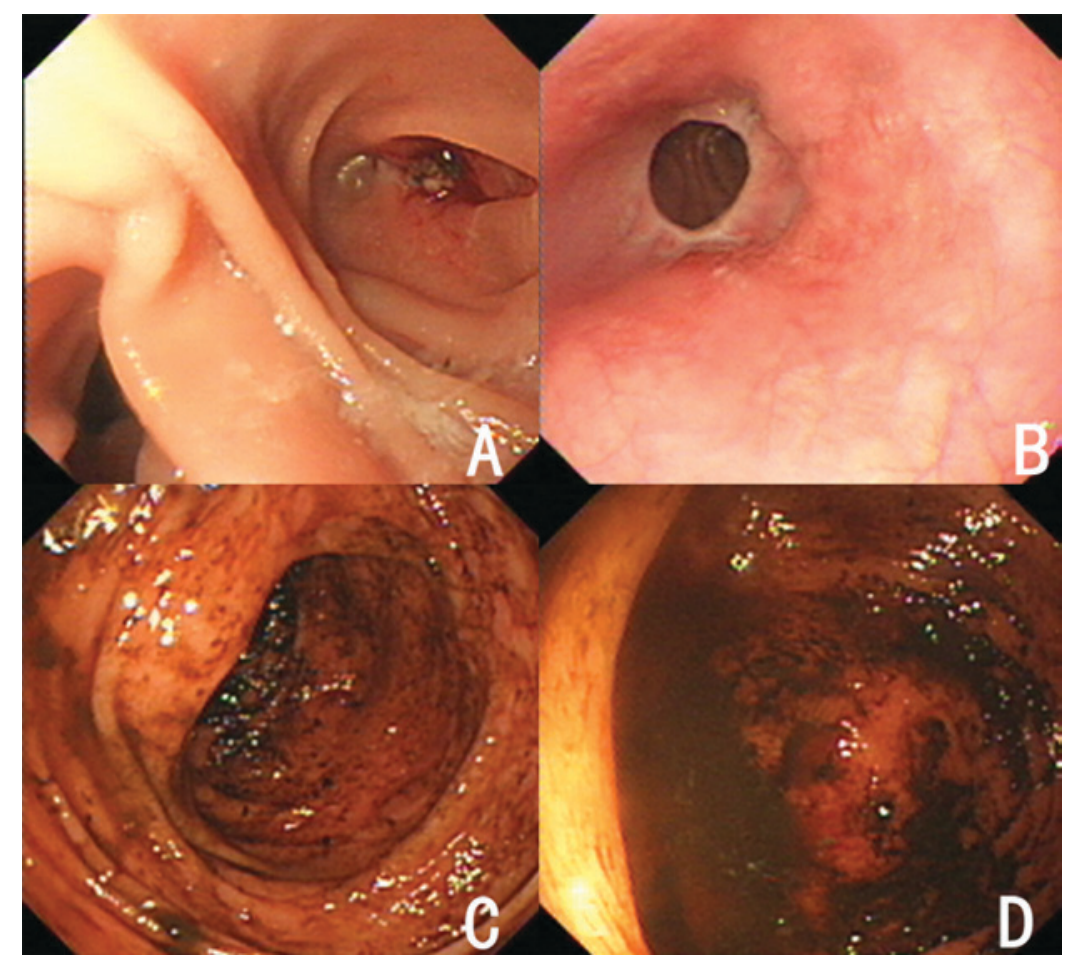

Figure 1. Endoscopy images from obtained on day 2 of hospitalization. (A) No blood was seen at the input and output loop; (B) no blood was seen at anastomosis; (C) remote hemorrhage was seen at the ascending colon; (D) remote hemorrhage was seen at the ileocecal valve.

58 beats per minute and a blood pressure of $120 / 80 \mathrm{mmHg}$ The patient was conscious without any signs of peritoneal irritation or Murphy's sign, and bowel sounds were normal. Routine blood test indicated hemoglobin $(\mathrm{Hb})$ levels of $97 \mathrm{~g} / \mathrm{l}$. Gastrointestinal endoscopy indicated no hemorrhagic spots (Fig. 1) and there was no hematochezia within 3 days after admission.

On March 16, 2012, the patient had intermittent hematochezia of up to $3,000 \mathrm{ml}$ and his blood pressure dropped to as low as $60 / 30 \mathrm{mmHg}$. Treatment by blood volume supplementation, hemostasis and blood transfusion proved ineffective, and digital subtraction angiography (DSA; Siemens AG, Munich, Germany) on March 17 revealed the development of a gastroduodenal artery stump (Fig. 2A). A microcatheter (Progreat; Terumo Corp., Tokyo, Japan) was subsequently inserted into the artery after super selection and dozens of absorbable gelatin sponges (Jinling Pharmaceutical Ltd., Nanjing, China) were used on their own for embolization to prevent gastrointestinal infarction, which was found successful based on angiography (Fig. 2B).

Six h later, the patient had massive hematochezia again of about 4,000 $\mathrm{ml}$, and $\mathrm{Hb}$ levels were as low as $32 \mathrm{~g} / \mathrm{l}$. After multidisciplinary discussion, surgeons did not recommend an additional operation. DSA was performed again on March 18, which revealed bleeding in the same location (Fig. 2C). A total of 27 micro-coil springs (Beijing Cook Medical Equipment Co., Ltd., Beijing, China) and $>20$ gelatin sponges were used to perform CHA embolization based on re-examination (Fig. 2D).

The patient underwent transfusion of red blood cells and other blood products (nearly $8,000 \mathrm{ml}$ ) within $48 \mathrm{~h}$. Two days after embolization, severe liver function damage, endogenous Escherichia coli blood infection, urinary tract infection, pulmonary infection and right pleural effusion occurred sequentially (Fig. 3). The pathological examination was completed using hemotoxylin and eosin staining (H\&E) to assess pleural effusion and no tumor cells were observed (Fig. 4). The patient was discharged from hospital after a 15-day course of support nutrition therapy and antibiotic treatment including moxifloxacin, cefatriaxone, meropenem, as well as right pleural effusion drainage $(500 \mathrm{ml})$, with no malignant cells.

From April 17, 2012, the patient began to show intermittent rigors and fever, and his body temperature rose to $39.4^{\circ} \mathrm{C}$, which was associated with frequent and urgent urination and odynuria. A urine routine test revealed 2+ leukocyte levels and a procalcitonin (PCT) level of $3.74 \mathrm{ng} / \mathrm{ml}$, which was indicative of a urinary system infection. After 3 days of antibiotic treatment with ciprofloxacin, the patient's body temperature further increased to $40.0^{\circ} \mathrm{C}$, and he was admitted to the hospital again on April 20.

Blood, urine and stool specimens were obtained from the patient on admission to perform bacterial and fungal culture tests. Four sets of venous blood specimens (10 $\mathrm{ml}$ per bottle) were aseptically collected from bilateral arms of the patient during chills and fever. The specimens were rapidly injected into special blood culture bottles, mixed immediately and submitted for detection. To culture and isolate pathogenic microorganisms, the blood culture bottles were incubated in the BacT/Alert 3D automated blood culture instrument (Organon Teknika LLC., Durham, NC, USA).

When the instrument alarm turned positive, the culture was immediately transferred to blood agar plates, a MacConkey agar plate and chocolate agar plates for bacterial culture. The culture was also inoculated to Sabouraud dextrose agar (SDA) and potato dextrose agar (PDA) for fungal culture. For 


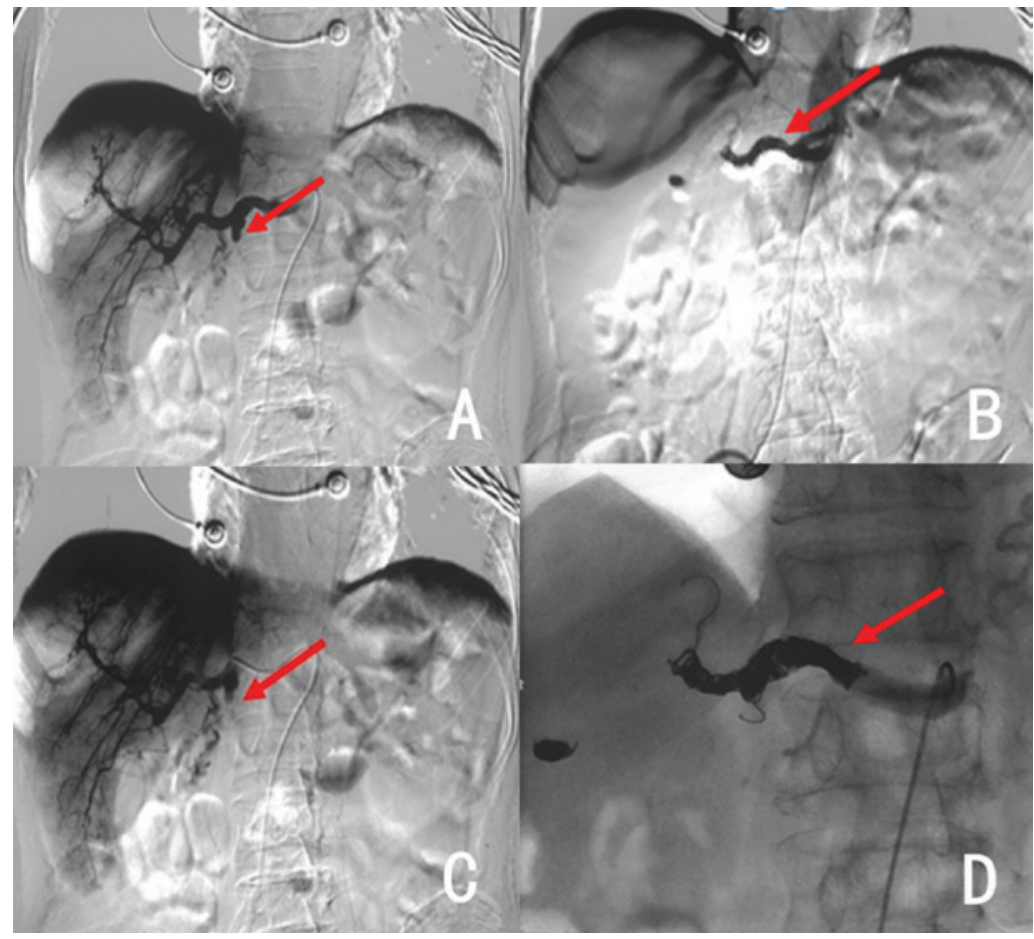

Figure 2. Changes in angiography prior to and after two embolizations. (A) Extravasation of contrast agent in the gastroduodenal artery stump (day 5 of hospitalization); (B) angiography revealing complete embolization without extravasation of the contrast agent (day 5 of hospitalization); (C) extravasation of the contrast agent in the gastroduodenal artery stump (day 6 of hospitalization); (D) location of the embolic coil spring: Common hepatic artery (day 6 of hospitalization).

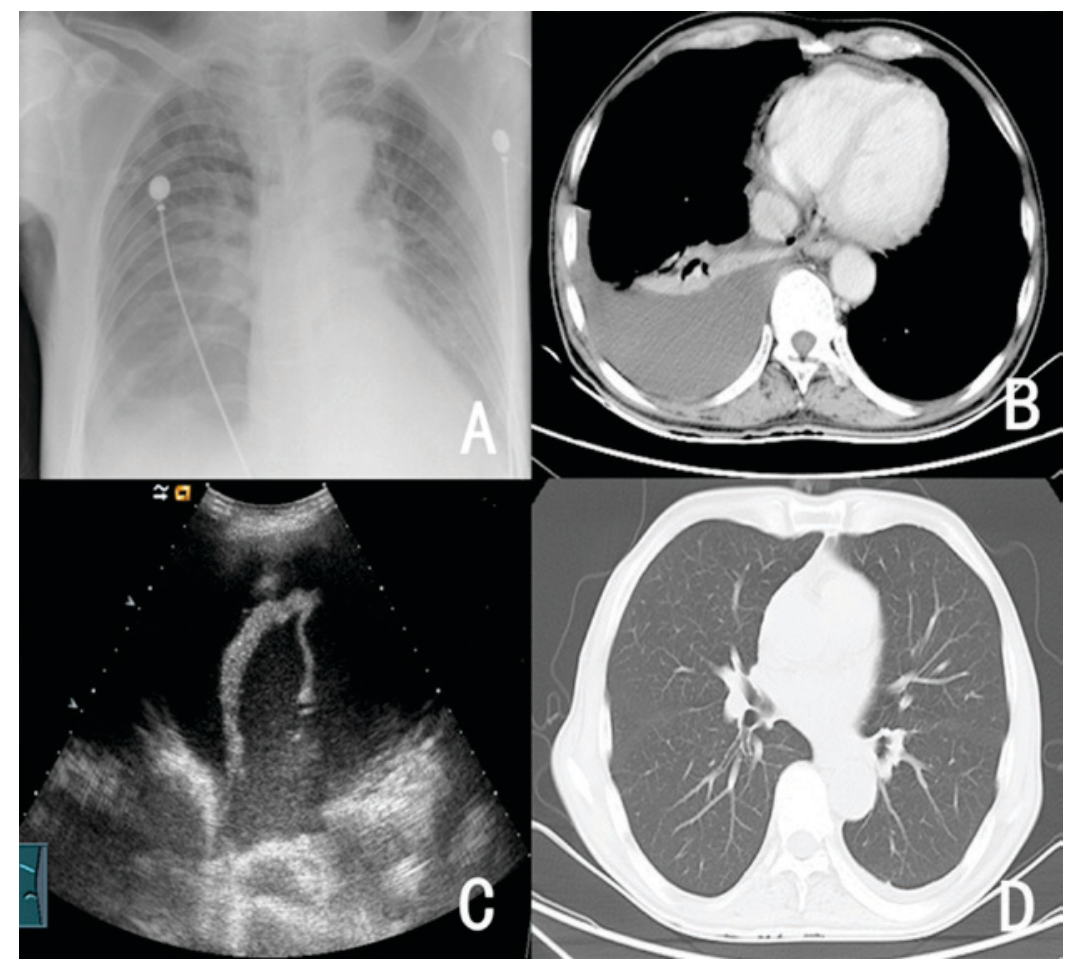

Figure 3. Imaging exam after embolism. (A) Bedside chest radiography revealing patchy shadow in the right lower lung and consolidated lung tissue margin in the outer zone of the right lower lung (day 4 of second embolization); (B) chest CT scan showing high-density pleural effusion and right lower lobe consolidation (day 14 of second embolization); (C) ultrasound scan revealing pleural effusion at a maximum depth of $11.2 \mathrm{~cm}$ with a weak echo (day 14 of second embolization); (D) chest CT scan demonstrating no obvious inflammatory manifestations (day 46 of second embolization). CT, computed tomography.

bacteriological examination, Gram staining and microscopic examination were performed, and the preliminary results were provided to the clinicians. Fungal morphology was examined using the steel ring method, and the culture was grown on PDA plates at $25^{\circ} \mathrm{C}$ for 7 days, followed by lactic acid-phenol-cotton blue staining. 
Table I. Changes in indicators of infection at different exam dates.

\begin{tabular}{lccccccc}
\hline Item & Normal value & D1 & D5 & D8 & D11 & D15 & D20 \\
\hline WBC $\left(10^{9} / \mathrm{l}\right)$ & $3.5-9.5$ & 7.7 & 5.69 & 6.14 & 10.86 & 7.41 & 7.57 \\
Neutrophils $(\%)$ & $40-75$ & 65.7 & 63.7 & 54.9 & 85.3 & 67.7 & 71.5 \\
Hemoglobin $(\mathrm{g} / \mathrm{l})$ & $130-175$ & 85 & 74 & 82 & 78 & 78 & 78 \\
Platelets $\left(10^{9} / \mathrm{l}\right)$ & $125-350$ & 208 & 143 & 290 & 264 & 269 & 225 \\
CRP $(\mathrm{mg} / \mathrm{l})$ & $<3$ & 144 & 45 & 17 & 66 & 30 & 41 \\
G $(\mathrm{pg} / \mathrm{ml})$ & $<100.5$ & ND & ND & ND & 62.3 & 131.8 & ND \\
GM $(\mathrm{ng} / \mathrm{ml})$ & $<0.5$ & ND & ND & ND & 4.66 & 0.16 & ND \\
PCT $(\mathrm{ng} / \mathrm{ml})$ & $<0.25$ & 3.74 & 9.45 & ND & 72.67 & 4.04 & ND
\end{tabular}

ND, not determined; WBC, white blood cells; CRP, C-reactive protein; PCT, procalcitonin; D, day; G, 1,33-dextran; GM, galacto-mannan of Aspergillus antigens. ${ }^{\mathrm{a}} \mathrm{Admission}$ day and ${ }^{\mathrm{b}} \mathrm{day} 1$ of Voriconazole.

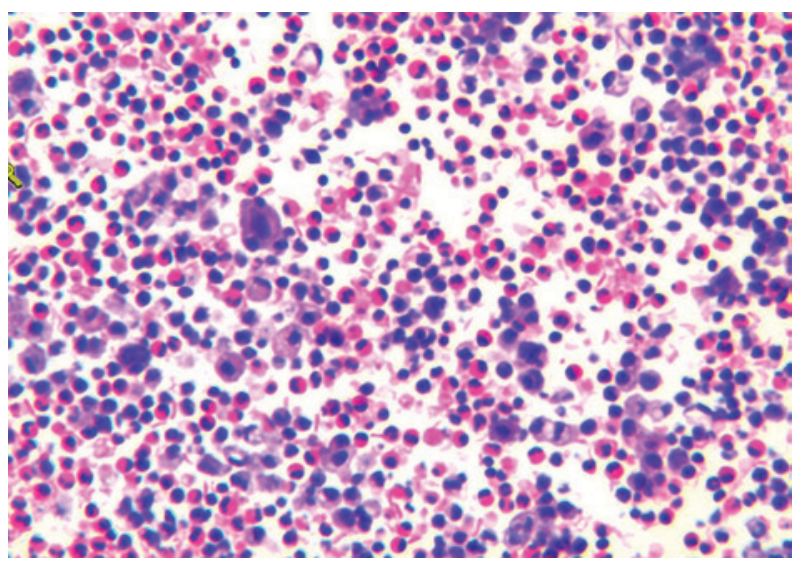

Figure 4. Pathology of pleural fluid. Microscopic examination revealing the presence of neutrophils, lymphocytes, eosinophils and proliferative mesothelial cells without malignant cells (day 16 of second embolization; at a magnification of $\times 10, \mathrm{H} \& \mathrm{E})$.

After collecting bacterial samples, the patient was given meropenem, and his body temperature returned to normal 2 days later, with negative blood, urine and stool analysis results. The treatment was continued until April 26, when the medication was replaced with cefuroxime sodium $(1.5 \mathrm{~g}$ b.i.d). On April 30, the fever without chilling occurred again with a maximum temperature of $39.6^{\circ} \mathrm{C}$. Chemical examination revealed high-sensitivity $\mathrm{C}$-reactive protein (CRP) of $66 \mathrm{mg} / 1, \mathrm{PCT}$ of $72.67 \mathrm{ng} / \mathrm{ml}$ and galacto-mannan of Aspergillus antigens of $4.66 \mathrm{ng} / \mathrm{ml}$. On the same day, only fungus was seen in blood culture samples obtained at different time-points on admission and from different parts of his upper limbs. No bacteria were detected either by direct microscopic observation or culture examination upon instrument alert.

Identification of pathogenic fungus was according to morphological characteristics: i) Microscopic examination: After instrument alarm, blood culture was extracted from the bottle with a syringe; hyaline and $45^{\circ}$-branching hyphae were seen on direct smear; ii) Culture examination: The colonies grew fast on SDA medium and developed a black powder surface; under a microscopic view, conidial heads consisting

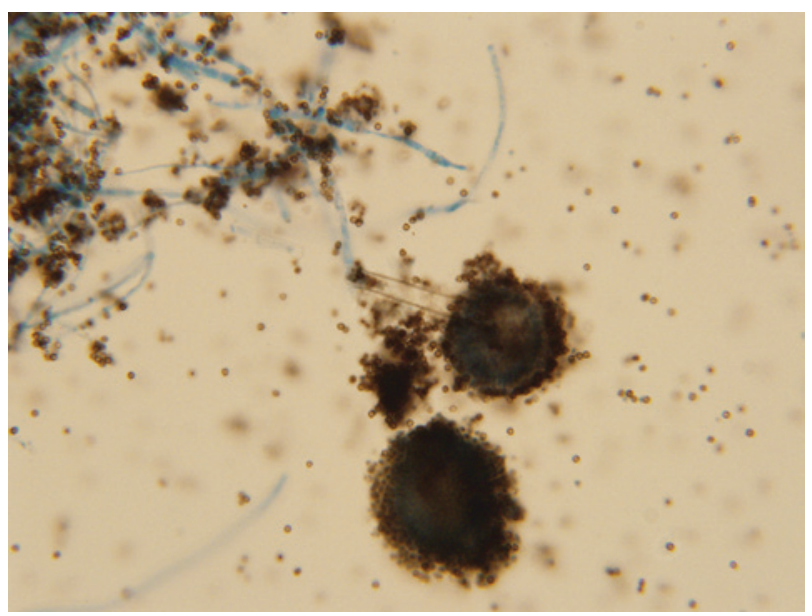

Figure 5. Microphotograph of Aspergillus niger. Conidial heads consisting of spherical vesicles, globose or subglobose were visible; the entire spherical vesicle was covered with two layers of sterigma, one layer thick and the other layer short; the sterigma was radially arranged, black and bearing chains of spores (magnification, $\mathrm{x} 10$ ).

of spherical vesicles, globose or subglobose, were visible; the entire spherical vesicle was covered with two layers of sterigma, one layer thick and the other layer thin; the sterigma was radially arranged, black and bore chains of spores (Fig. 5). According to the above characteristics, the fungus was identified as $A$. niger.

An anti-fungal drug sensitivity test against four types of anti-fungal drugs was performed. The minimum inhibitory concentrations for the fungus were as follows: Voriconazole $0.25 \mu \mathrm{g} / \mathrm{ml}$, amphotericin B $4.0 \mu \mathrm{g} / \mathrm{ml}$, itraconazole $0.5 \mu \mathrm{g} / \mathrm{ml}$ and caspofungin $0.06 \mu \mathrm{g} / \mathrm{ml}$.

After Aspergillus BSI was diagnosed, intravenous therapy with voriconazole (400 mg bid d1, $200 \mathrm{mg}$ bid d2-d14) was performed. The patient's body temperature returned to normal 2 days later, PCT fell to $4.04 \mathrm{ng} / \mathrm{ml} 6$ days later, and other inflammatory markers fell significantly as well (Table I). Two weeks later, sequential therapy with oral voriconazole (200 mg bid) was applied for 4 weeks. At the two-year follow-up on July 2015, no evidence of recurrent fungal BSI, gastrointestinal bleeding or metastatic relapse was identified. 
The patient provided written informed consent regarding the publication of his data and images in the present study.

\section{Discussion}

Selective arterial embolization is an important treatment for gastrointestinal bleeding of unknown primary origin (6-8). Superselection of the bleeding arteries is mostly used in the clinic (9-11), but embolization of the CHA is rarely used, since it may severely damage liver function (12). Gastrointestinal bleeding in the present case was likely due to digestive system reconstruction after total gastrectomy and duodenal residual corrosion by digestive juice (e.g., gastric acid bile and pancreatin), which led to mucosal damage and ulceration, which may have also been aggravated by the adverse reactions of nausea and vomiting following chemotherapy. All of the above mentioned factors may have led to the rupture of the vascular branch of the gastroduodenal artery supplying residual duodena to cause gastrointestinal bleeding.

In addition, possible vascular malformation was caused by the change in anatomical structure after surgery, and it later developed into a pseudoaneurysm (13-16), which ruptured and bled massively after systemic chemotherapy. All factors contributed to the bleeding, resulting in a life-threatening condition. Surgical operation at this juncture is extremely risky, and gastroduodenal artery bleeding was diagnosed twice using DSA. The first attempt of embolization failed, likely as a result of the fact that the bleeding artery was close to the CHA, and the rapid blood flow led to re-canalization. The second embolization was performed on the CHA using permanent embolic micro-coil springs in addition to gelatin sponges, which stopped the bleeding and saved the patient's life. Gelatin sponge particles may be absorbed in the short term, and if the bleeding recurs, the use of gelatin sponge particles alone should be avoided due to permanent embolism. CHA embolization changed the blood supply of the liver and caused severe embolism syndrome, which was later cured by liver protection and anti-biotic treatment.

The patient presented with chill, fever and urinary irritation 1 month after embolization, which was probably due to a urinary infection. The influence of the contrast agent during continuous DSA could not be ruled out. The fever recurred during anti-biotic therapy and blood culture indicated growth of A. niger, which is rarely seen in the clinic. Caused mostly by Candida and occasionally by Cryptococcus neoformans $(2,17)$, fungemia has a poor prognosis, and it is usually an end-stage symptom of an immunocompromised patient. Aspergillus grows naturally, and the majority of reported Aspergillus BSIs are induced by exogenous infection due to large-area burns $(18,19)$. Invasive Aspergillus infection generally occurs in the lungs in the early stages, and then spreads to the central nervous system and other organs. However, it seldom enters the blood and Aspergillus BSI is therefore rare in the clinic.

Imageological examination of the patient indicated no translocation of Aspergillus infection, and therefore, the cause of the A. niger BSI remains unknown. The following factors are potential causes for the infection: i) Test contamination: A. niger was not detected in any of the multiple blood samples submitted on the same day, so test contamination is excluded; ii) blood sample contamination during collection: Two blood samples with positive results were collected from different parts of the body at different time-points by different nurses, so blood sample contamination during collection is excluded; ii) low immunity: Surgery, post-operative chemotherapy, massive hemorrhage of the gastrointestinal tract, severely damaged liver function after CHA embolization and BSI with Gram-negative bacteria, as well as the application of strong anti-biotics, may all significantly lower immunity and result in exogenous Aspergillus entering the blood and cause infection; and iv) invasive operation contamination: The patient received invasive DSA and embolization twice within $24 \mathrm{~h}$, and the embolization required various embolic materials. As the only invasive operation with a long exposure duration in the course of the disease, embolization of the CHA cannot be totally excluded as the cause underlying A. niger BSI, even though there was an extended time period between the operation and infection, and none of the other patients undergoing interventional therapy within 1 week prior to and after the patient were diagnosed with A. niger BSI.

Within 3-4 months after gastric cancer surgery, the patient had successive massive hemorrhage of the gastrointestinal tract and A. niger BSI, which are critical illnesses, and he was cured by $\mathrm{CHA}$ embolization and anti-fungal therapy with voriconazole. Serious infections such as those with Aspergillus tend to occur in patients with hematological diseases and cancer who are immunocompromised (20-22). It is important to provide active protection in order to prevent infection during invasive tests and treatments. In addition, it is worth noting that Aspergillus culture takes a long time by continuous passage culture (23). Thus, D-glucose, GM, PCT and CRP assessments should be combined to achieve a timely anti-fungal treatment.

\section{References}

1. Rosa C, Araujo R, Rodrigues AG, Pinto-de-Sousa MI and Pina-Vaz C: Detection of Aspergillus species in BACTEC blood cultures. Med Microbiol 60: 1467-1471, 2011.

2. Arendrup MC, Fuursted K, Gahrn-Hansen B, Jensen IM, Knudsen JD, Lundgren B, Schønheyder HC and Tvede M: Seminational surveillance of fungemia in Denmark: Notably high rates of fungemia and numbers of isolates with reduced azole susceptibility. J Clin Microbiol 43: 4434-4440, 2005.

3. Person AK, Chudgar SM, Norton BL, Tong BC and Stout JE: Aspergillus niger: An unusual cause of invasive pulmonary aspergillosis. J Med Microbiol 59: 834-838, 2010.

4. Ali S, Malik A, Bhargava R, Shahid M and Fatima N: Aspergillus colonization in patients with bronchogenic carcinoma. Asian Cardiovasc Thorac Ann 22: 460-464, 2014.

5. Gifford AH, Lahey T and Fordham Von Reyn C: Fatal hemoptysis from invasive Aspergillus niger in a patient with cavitary lung disease and Mycobacterium avium complex infection. Med Mycol 44: 557-560, 2006.

6. Renzulli P, Candinas D and Seiler CA: Acute 'difficult' gastrointestinal bleeding. Ther Umsch 63: 311-319, 2006.

7. Remy-Jardin M, Bouaziz N, Dumont P, Brillet PY, Bruzzi J and Remy J: Bronchial and nonbronchial systemic arteries at multi detector row CT angiography: Comparison with conventional angiography. Radiology 233: 741-749, 2004.

8. Boufi M, Hashemi AA, Azghari A, Hartung O, Ramis O, Moutardier V and Alimi YS: Endovascular management of severe bleeding after major abdominal surgery. AnnVasc Surg 27: 1098-1104, 2013.

9. Lee JH, Hwang DW, Lee SY, Hwang JW, Song DK, Gwon DI, Shin JH, Ko GY, Park KM and Lee YJ: Clinical features and management of pseudoaneurysmal bleeding after pancreatoduodenectomy. Am Surg 78: 309-317, 2012. 
10. Li Z, Jie Z, Liu Y and Xie X: Management of delayed hemorrhage following radical gastrectomy for gastric carcinoma patients. Hepatogastroenterology 59: 2016-2019, 2012.

11. Anil G, Tan AG, Cheong HW, Ng KS and Teoh WC: Emergency gastroduodenal artery embolization by sandwich technique for angiographically obvious and oblivious, endotherapy failed bleeding duodenal ulcers. Clin Radiol 67: 468-475, 2012.

12. Sanada Y, Kondo H, Goshima S, Kanematsu M, Tanaka Y, Tokuyama Y, Osada S and Yoshida K: Liver abscess after common hepatic artery embolization for delayed hemorrhage following pancreaticoduodenectomy: A case report. Case Rep Med 2010: 280430, 2010.

13. Lee HG, Heo JS, Choi SH and Choi DW: Management of bleeding from pseudoaneurysms following pancreaticoduodenectomy. World J Gastroenterol 16: 1239-1244, 2010.

14. Loffroy R and Guiu B: Arterial embolization is the best treatment for pancreaticojejunal anastomotic bleeding after pancreatoduodenectomy. World J Gastroenterol 15: 4090-4091, 2009.

15. Nonokuma M, Okazaki M, Higashibara H, Kimura S, Kora S, Urakawa H, Shinagawa Y, Osame A, Ueki T and Nakayama T: Successful embolization of pancreaticoduodenal artery pseudoaneurysm in a patient with common hepatic arterial occlusion after modified pancreatoduodenectomy with preservation of arteries in the head of pancreas. Hepatogastroenterology 56: 245-248, 2009.

16. Santoro R, Carlini M, Carboni F, Nicolas C and Santoro E: Delayed massive arterial hemorrhage after pancreaticoduodenectomy for cancer. Management of a life-threatening complication. Hepatogastroenterology 50: 2199-2204, 2003.
17. Guy GE, Shetty PC, Sharma RP, Burke MW and Burke TH: Acute lower gastrointestinal hemorrhage: Treatment by superselective embolization with polyvinyl alcohol particles. AJR Am J Roentgenol 159: 521-526, 1992.

18. Shinohara MM, Miller CJ and Seykora JT: Pigmented fruiting bodies and birefringent crystals in a surgical wound: A clue to Aspergillus niger infection. J Cutan Pathol 38: 603-606, 2011.

19. Singhal P, Usuda K and Mehta AC: Post-lung transplantation Aspergillus niger infection. J Heart Lung Transplant 24: 1446-1447, 2005.

20. Fianchi L, Picardi M, Cudillo L, Corvatta L, Mele L, Trapè G, Girmenia $C$ and Pagano L: Aspergillus niger infection in patients with haematological diseases: A report of eight cases. Mycoses 47: 163-167, 2004.

21. Aksoy DY, Turker A, Altundag MK, Abali H, Durusu M, Erman M, Uner A, Sungur AA, Unal S and Uzun O: Concomitant Mycobacterium tuberculosis and Aspergillus niger infection in a patient with acute myeloid leukemia. Chemotherapy 49: 264-266, 2003.

22. Vermeulen E, Maertens J, Meersseman P, Saegeman V, Dupont L and Lagrou K: Invasive Aspergillus niger complex infections in a Belgian tertiary care hospital. Clin Microbiol Infect 20: O333-O335, 2014.

23. Garcia-Vidal C and Carratalà J: Pathogenesis of invasive fungal infections. Enferm Infecc Microbiol Clin 30: 151-158, 2012 (In Spanish). 